DRAFT VERSION NOVEMBER 4, 2018

Preprint typeset using LTEX style emulateapj v. 5/2/11

\title{
EXPLORING HALO SUBSTRUCTURE WITH GIANT STARS: SUBSTRUCTURE IN THE LOCAL HALO AS SEEN IN THE GRID GIANT STAR SURVEY INCLUDING EXTENDED TIDAL DEBRIS FROM $\omega$ CENTAURI
}

\author{
SteVen R. Majewski ${ }^{1}$, David L. Nidever ${ }^{1}$, Verne V. SMith ${ }^{2}$, Guillermo J. Damke ${ }^{1}$, \\ William E. KunKel ${ }^{3}$, Richard J. PATterson ${ }^{1}$, DMitry BIZYAEV ${ }^{4}$, ANd Ana E. García PÉREZ ${ }^{1}$ \\ Draft version November 4, 2018
}

\begin{abstract}
We present the latitude-normalized radial velocity $\left(v_{b}\right)$ distribution of 3318 subsolar metallicity, $V \lesssim 13.5$ stars from the Grid Giant Star Survey (GGSS) in Southern Hemisphere fields. The sample includes giants mostly within $\sim 5 \mathrm{kpc}$ from the Galactic disks and halo. The nearby halo is found to (1) exhibit significant kinematical substructure, and (2) be prominently represented by several velocity coherent structures, including a very retrograde "cloud" of stars at $l \sim 285^{\circ}$ and extended, retrograde "streams" visible as relatively tight $l-v_{b}$ sequences. One sequence in the fourth Galactic quadrant lies within the $l-v_{b}$ space expected to contain tidal debris from the "star cluster" $\omega$ Centauri. Not only does $\omega$ Cen lie precisely in this $l-v_{b}$ sequence, but the positions and $v_{b}$ of member stars match those of $N$-body simulations of tidally disrupting dwarf galaxies on orbits ending with $\omega$ Cen's current position and space motion. But the ultimate proof that we have very likely found extended parts of the $\omega$ Cen tidal stream comes from echelle spectroscopy of a subsample of the stars that reveals a very particular chemical abundance signature known to occur only in $\omega$ Cen. The newly discovered $\omega$ Cen debris accounts for almost all fourth Galactic quadrant retrograde stars in the southern GGSS, which suggests $\omega$ Cen is a dominant contributor of retrograde giant stars in the inner Galaxy.
\end{abstract}

Subject headings: Galaxy: structure — galaxies: kinematics and dynamics — galaxies: interactions — galaxies: individual ( $\omega$ Centauri)

\section{LOCAL RETROGRADE HALO TIDAL STREAMS}

If the Galactic halo has been formed partly (Searle \& Zinn 1978) or entirely (e.g., Majewski 1993) from accretion of smaller systems then one might expect groups of stars with halo-like motions having strong velocity coherence (e.g., Helmi \& White 1999; Meza et al. 2005) near the Sun. Claims for nearby halo moving groups date back at least to Eggen \& Sandage (1959) and include several retrograde candidates including, in particular, the widely recognized Kapteyn Group - among the halo groups long discussed by Eggen (e.g., 1965 1996a b). Majewski et al. (1994, 1996) analyzed nearby halo stars (selected by asymmetric drift) towards the North Galactic Pole (NGP) and claimed strong organization of their phase space distribution into a few clumps, including a prominent retrograde moving group initially identified via proper motions in Majewski (1992). A possible association of horizontal branch stars to this retrograde feature has been identified by Kinman et al. (2007). Eggen (1996b) suggests an association of the Majewski (1992) retrograde group to Kapteyn's group.

More recently, interest in the notion that the globular cluster $\omega$ Centauri (" $\omega$ Cen") may be the remnant core of a tidally disrupted satellite galaxy (e.g., Lee et al. 1999; Majewski et al. 2000b, Bekki \& Freeman 2003) - a notion inspired by (1) internal chemical and age distributions belying multiple $\omega$ Cen stellar populations, (2) the example of the similarly massive cluster M54 located in/near/as the "core" of the dis-

\footnotetext{
${ }^{1}$ Dept. of Astronomy, University of Virginia, Charlottesville, VA 22904-4325 (srm4n, dln5q, gjd3r, ricky, aeg4x@virginia.edu)

2 National Optical Astronomy Observatories, PO Box 26732, Tucson, AZ 85726 (vsmith@noao.edu)

${ }^{3}$ Las Campanas Observatory, Casilla 601, La Serena, Chile (kunkel@jeito.lco.cl)

${ }^{4}$ New Mexico State University/Apache Point Observatory, Sunspot NM 88349 (dmbiz@apo.nmsu.edu)
}

rupting Sagittarius galaxy (Majewski et al.2000b) and apparent chemical similarities of M54+Sagittarius to $\omega$ Cen (Carretta et al.2010), and (3) the unusual, low-inclination, retrograde orbit of $\omega$ Cen itself (Dinescu 2002) — has led to several $\omega$ Cen tidal disruption simulations; these models generally produce retrograde-moving, $\omega$ Cen debris relatively near the solar circle (Dinescu 2002, Tsuchiya et al. 2003, 2004; Mizutani et al. 2003; Bekki \& Freeman 2003). This has prompted searches for retrograde halo stars possibly shed by the "cluster" and led to suggestions that an " $\omega$ Cen" signal is present among local metal-weak stars (Dinescu 2002; Mizutani et al. 2003; Meza et al. 2005). In fact, Eggen (e.g., 1978) speculated a connection between his Kapteyn's star group and $\omega$ Cen (see also Kotoneva et al. 2005), a dubious connection (Proust \& Foy 1988) before the breadth of $\omega$ Cen's metallicity distribution function was fully recognized, but more recently bolstered by detailed chemical analysis of Kapteyn group stars (Wylie-de Boer et al.2010).

Despite these recent claims for stripped $\omega$ Cen stars in the solar neighborhood, searches for extratidal stars near $\omega$ Cen itself have been less promising. While the photometric search by Leon et al. (2000) seemed to suggest a "significant" pair of tidal tails extending from $\omega$ Cen, these results were cast in doubt when the substantial foreground differential reddening was assessed (Law et al. 2003) 5 An expansive spectroscopic search for $\omega$ Cen stars beyond its tidal radius by Da Costa \& Coleman (2008) reveals only six candidates among more than 4,000 stars selected from the $\omega$ Cen giant branch in the color-magnitude diagram; these authors suggest that this meager haul is consistent with models where most stripping took place long ago, and with the lost stars now widely distributed about the Galaxy. Stronger support for the tidally-disrupted dwarf galaxy model, and for confidently linking solar neigh-

\footnotetext{
5 Leon et al. (2000) themselves warned that dust extinction might be influencing their results.
} 
borhood candidate members with $\omega$ Cen itself, would come from actually being able to trace debris along the satellite's orbit, and, eventually, from $\omega$ Cen continuously to the solar neighborhood.

Here we report detection of a kinematically coherent "tidal debris" signature spanning $\gtrsim 60^{\circ}$ of Galactic longitude in a large radial velocity $(\mathrm{RV})$ survey of giant stars mostly $(\sim$ $95 \%$ ) within $\sim 5 \mathrm{kpc}$ of the Sun. Stars within this dynamically coherent group show a specific chemical marker thought to be unique to $\omega$ Cen, as well as distances and velocities consistent with models of $\omega$ Cen tidal debris. Though still mostly only a few kiloparsecs away, these extended tidal debris stars provide a start at tracing the $\omega$ Cen stream and provide crucial, though still crude, dynamical constraints on the disruption of the closest known dwarf galaxy to the Sun. At minimum, these discovered debris stars suggest that $\omega$ Cen may be a principal source of local retrograde stars.

\section{SUBSTRUCTURE IN THE SOUTHERN GGSS}

Our analysis uses the Grid Giant Star Survey (GGSS), a partially-filled, all-sky search for giant stars using the Washington $M, T_{2}+D D O 51$ photometric selection technique described in Majewski et al. (2000a). The GGSS had as one goal the identification of bright but distant, metal-poor giants suitable for the Astrometric Grid of the (now defunct) Space Interferometry Mission. To this end, a specific subset of potential SIM Grid stars was drawn from the GGSS: the four most distant giant stars with $M<13.5$ in each of the 1302 evenly spaced, $\sim 0.4-0.6 \mathrm{deg}^{2}$ GGSS fields, where photometric distances were estimated by assigning absolute magnitudes to stars from their position in the $\left(M-T_{2}, M-D D O 51\right)$ diagram (which separates dwarf and giant stars and, within these luminosity classes, sorts by metallicity; Majewski et al. 2000a). This particular selection biases this "SIM Grid sample" to more metal poor giants, because these are farther at a given apparent magnitude. An echelle resolution study of 774 Grid candidates selected in this way verified $100 \%$ of them to have giant branch surface gravities, and indicated a median $[\mathrm{Fe} / \mathrm{H}] \sim-0.7$ and distance of $\sim 2 \mathrm{kpc}$ (Bizyaev et al. 2006), but with long tails to more distant and metal-poor stars. Thus, the sample contains a mix of old thin disk, Intermediate Population II (IPII) thick disk and a smaller fraction of halo stars in mean proportions varying by Galactic latitude. Our initial analysis here relies on $R \sim 2,600$ spectroscopy of a larger sample of 3318 GGSS "SIM Grid" giants from our southern observing campaign (sky distribution shown in Fig. 1a); further discussion of the GGSS is given in Patterson et al. (2001) and S. Majewski (in preparation).

In 1999 a spectroscopic follow-up campaign for these candidate Astrometric Grid stars began at Las Campanas Observatory using the Modular Spectrograph on the Swope 1-m telescope (and the DuPont 2.5-m for 68 stars). The typical set-up used the 600 line/mm grating to sample a $\sim 2000 \AA$ spectral region including both $\mathrm{H} \alpha$ and $\mathrm{H} \beta$ at $1.0 \AA$ pixel $^{-1}$ (in a small fraction of cases the $\mathrm{Ca}$ infrared triplet region was observed; details of both setups are in Majewski et al. (2004). The $S / N$ of the spectra exceeds 10 in almost all cases and reaches $>50$ in some cases. RV cross-correlation of the spectra used the methodology discussed in Majewski et al. (2004). Typical RV errors are $5-10 \mathrm{~km} \mathrm{~s}^{-1}$, estimated a variety of ways including repeat measures of many stars and comparison to echelle resolution RVs.

Figure 1b shows the distribution of Galactic Standard of

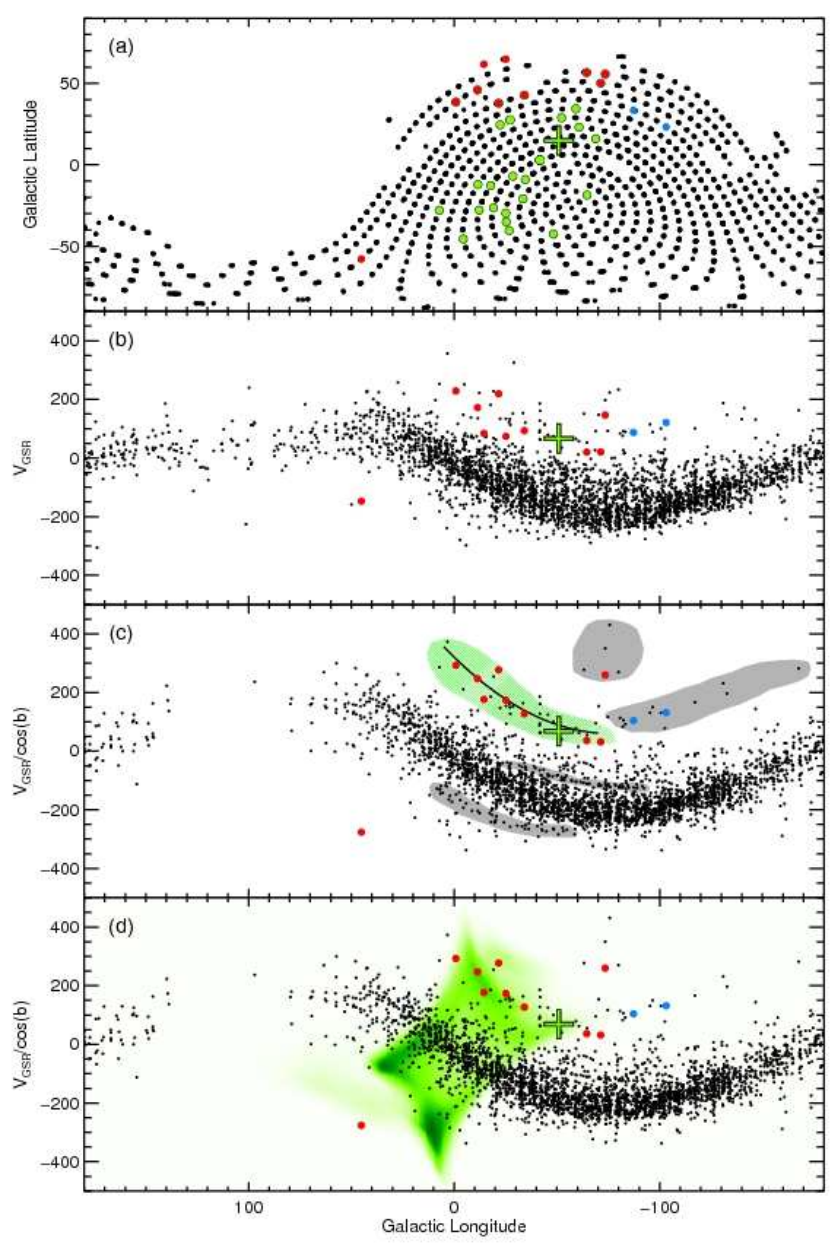

FIG. 1.- The distribution of the GGSS giant stars used in this study in (a) Galactic coordinates, (b) $v_{\mathrm{GSR}}$ versus longitude, and (c) $v_{b}=v_{\mathrm{GSR}} / \cos b$ versus longitude (for only $|b|<60^{\circ}$ in panel $\mathrm{c}$ ). The curve and green shading in (c) highlight the arc of stars we believe contains $\omega$ Cen debris. Stars in this sequence are marked with green or red points in panel (a). Grey shadings show several other potential halo substructures. The position of the $\omega$ Cen core in all panels is shown by the large green cross. Panel (d) is the same as panel (c) but showing the "probability distribution" of $\omega$ Cen tidal debris based on our suite of models. Superposed red and blue points in all panels represent stars having echelle spectra with red designating those stars that follow the $\omega \mathrm{Cen}[\mathrm{Ba} / \mathrm{Fe}]-[\mathrm{Fe} / \mathrm{H}]$ patterns and blue those that do not.

Rest (GSR) RVs $\left(v_{G S R}\right)$ for the "SIM Grid" stars, assuming a solar motion in right-handed Galactic coordinates of $(+10.0,+225.3,+7.2) \mathrm{km} \mathrm{s}^{-1}$. For stars moving predominantly in Galactic planar orbits (e.g., disk stars and putative $\omega$ Cen debris), the approximate "planar RV" (i.e., the observed RV the star would have were it on the Galactic equator) is given by $v_{b}=v_{G S R} / \cos (b)$ (this latitude normalization breaks down at high latitude, where a star's $Z$ motion dominates the observed RV, so Fig. 1c is limited to stars with $|b|<60^{\circ}$ ). In this planar projection stellar populations more clearly sort by their relative asymmetric drifts, and Figure 1c for the most part shows the expected longitudinal distribution of $v_{G S R}$ for a predominantly thin disk/IPII mix of stars.

However, stars not following disk kinematics are evident, including a number having retrograde velocities. Among stars with halo-like velocities (and particularly among likely retrograde stars), outlier groupings (e.g., at $\left[l, v_{b}\right] \sim\left[280^{\circ}, 300\right.$ $\left.\mathrm{km} \mathrm{s}^{-1}\right]$ ) or thin, coherent strands of stars (e.g., from $\left[20^{\circ}\right.$, $-125 \mathrm{~km} \mathrm{~s}^{-1}$ ] to $\left[300^{\circ},-275 \mathrm{~km} \mathrm{~s}^{-1}\right]$ ) can be seen in Fig- 
ures $1 \mathrm{~b}$ and/or $1 \mathrm{c}$ (where we have highlighted some interesting features with shading). Such cold and coherent RV trends with sky position are characteristic of long tidal streams, such as the Sagittarius system (e.g., Majewski et al. 2004, Law et al. 2005); in this case, however, the substructure is found among relatively nearby giants and therefore corresponds to stars with a much broader sky distribution than typical for more distant streams (Fig. 1a). Such substructure among the GGSS giants is not surprising given that they probe distances similar to the mostly main sequence stars in the Majewski et al. (1994, 1996) study, which also showed significant halo substructure (but in only a single pencil beam). Moreover, a new "all-sky" study of bright $\mathrm{M}$ giants by A. Sheffield (in preparation) and probing comparable distances shows analogous, though even more striking, $\left[l, v_{b}\right]$ coherences among halo-like stars (most likely because $M$ giants probe typically younger tidal streams). Together, the GGSS giants, Sheffield M giants, Majewski et al. (1994, 1996) subdwarfs, and Kinman et al. horizontal branch stars point to the high degree of substructure in the halo even at the solar circle. Indeed, the degree of velocity coherence and substructure of the local halo does not differ much from that seen in the distant halo (Majewski 2004).

\section{TIDAL DEBRIS MODEL AND THE $\omega$ CENTAURI CONNECTION}

Are there stars in the GGSS sample that can be associated with $\omega$ Cen? The studies of solar neighborhood $\omega$ Cen debris mentioned in $\S 1$ were able to make use of the expected $\left(E, L_{z}\right)$ distributions to trawl for the best local representatives. Unfortunately, our giant stars are far enough away that most available proper motions (and therefore complete space velocity determinations) are unreliable. Therefore we winnow our search to those $\left[l, v_{b}\right]$ ranges expected to be populated by any realistic model of $\omega$ Cen tidal disruption. To do so we create a suite of N-body simulations of satellites undergoing tidal disruption along $\omega$ Cen-like orbits in the static Milky Way (MW) potential given by Johnston et al. (1995), with 30,000 particles representing the parent satellite in an initially Plummer configuration. To account for observational uncertainties that prohibit us from accurately knowing the true position, space motion, and therefore orbits of both $\omega$ Cen and the Sun, we create a grid of models spanning ranges of uncertainty around typical mean values for each critical interaction parameter (from Dinescu et al. 1999): $\omega$ Cen distances of [4.9,5.1,5.3] kpc, Galactic Cartesian velocities (right-handed system in the Galactic rest frame) of $V_{x}=[42,53,64,75,86] \mathrm{km} \mathrm{s}^{-1}, V_{y}=$ $[-52,-43,-34,-25,-16] \mathrm{km} \mathrm{s}^{-1}$, and $V_{z}=[-6,4,14] \mathrm{km} \mathrm{s}^{-1}$, a solar Galactocentric distance of $[7.0,7.75,8.5] \mathrm{kpc}$, and, to set the MW mass scale, a local circular velocity of [220,254] $\mathrm{km} \mathrm{s}^{-1}$, where the latter value is that suggested by Reid et al. (2009). Within this grid we adopt satellites of three different initial masses, [3e7, 3e8, 3e9] $\mathrm{M}_{\odot}$, and evolved for $\sim 0.4$ Gyr, ensuring that each model orbit places the satellite at the current $(l, b)$ position and RV of $\omega$ Cen.

These 4050 simulations produce a variety of tidal streams from which we can establish the range of possible $\left[l, v_{b}\right]$ distributions; in fact, the sum of these models (Fig. 1d) gives us something like a "probability distribution function" (PDF) of where the last 0.4 Gyr of $\omega$ Cen tidal debris might most likely lie (Fig. 1d). Comparing this to the GGSS distribution, and ignoring regions dominated by disk stars, we call attention to a particularly striking match of the PDF to a relatively tight sequence of likely retrograde stars over $l \sim 280-360^{\circ}$, high-

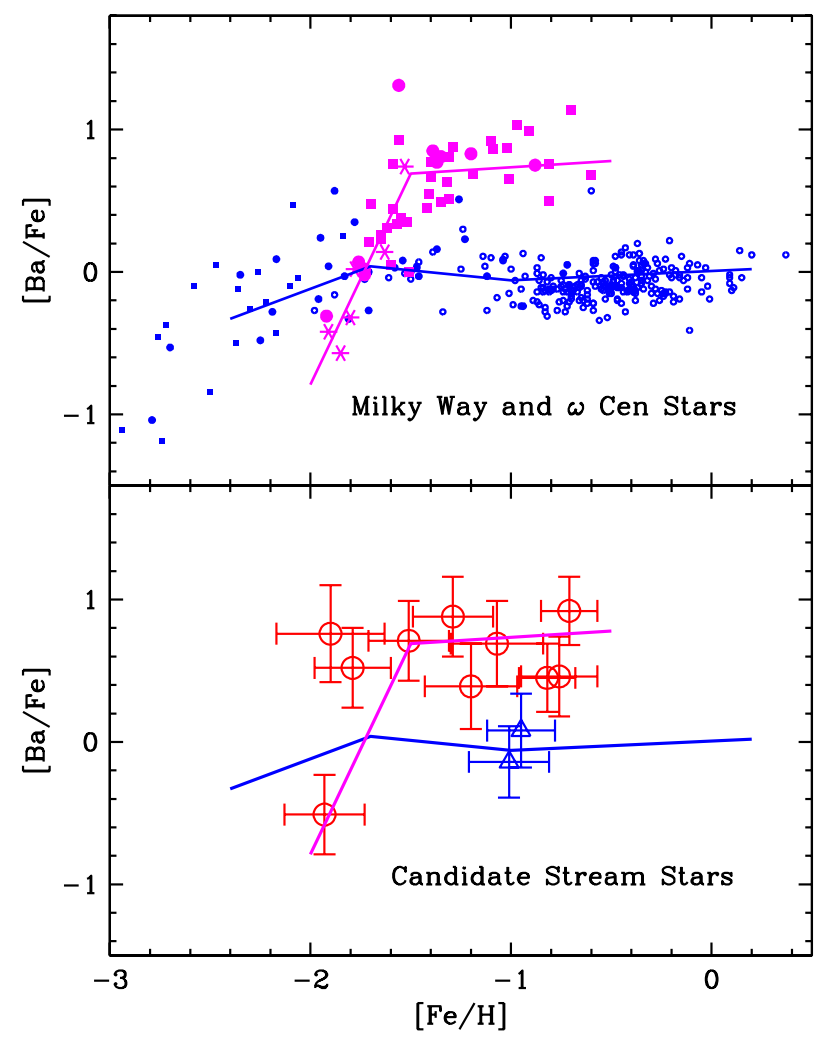

FIG. 2.- (Top panel) The distribution of $[\mathrm{Ba} / \mathrm{Fe}]-[\mathrm{Fe} / \mathrm{H}]$ for MW stars (blue points) and a characteristic locus (blue line) from data by Fulbright (2002), Johnson (2002), and Reddy et al. (2003 2006), overlaid with the same for $\omega$ Cen stars (magenta points and line) from Francois et al. (1988), Norris \& Da Costa (1995), and Smith et al. (2000). (Bottom panel) The distribution of barium abundances for the ten stars following the retrograde sequence lying within the " $\omega$ Cen PDF region" and containing the position of $\omega$ Cen shown in Fig. 1 (red points) versus those stars lying outside the " $\omega$ Cen PDF region" (blue). The colored lines are those shown in the top panel.

lighted with green shading in Figure 1c. That these stars form a coherent velocity structure orbiting in a near-Galactic planar orbit is demonstrated by the fact that, despite their broad sky distribution (green and red points, Fig. 1a), they show a coherent, string-like configuration in planar, $v_{b}$ projection.

A 2nd-order polynomial fit to the Figure 1c sequence with iterative rejection settles on 35 stars in the feature with an observed RV dispersion of only $\sim 40 \mathrm{~km} \mathrm{~s}^{-1}$. Most interestingly, the sequence passes through the $\left(l, v_{b}\right)$ position of $\omega$ Cen, also shown.

To test whether $\omega$ Cen stars might fall among those GGSS stars lying within this retrograde feature, high-resolution $(R \sim 55,000)$, echelle spectra for eight of them, as well as a control sample of four other halo GGSS stars (including two very extreme $v_{b}$ stars at similar longitudes), were obtained using the Sandiford echelle spectrometer (McCarthy et al. 1993) on the McDonald 2.1-m Struve telescope. Given the modest wavelength coverage and $S / N \sim 25-50$ of the spectra, iron abundances were derived from a set of unblended Fe I lines using measured equivalent widths. The stellar parameters $T_{\text {eff }}$ and $\log g$ were taken from the analysis of GGSS stars by Bizyaev et al. (2006), with determinations of the microturbulence velocities $(\xi)$ set by the Fe I lines measured for this study.

The well-defined Ba II line at $5854 \AA$ was used as an s- 


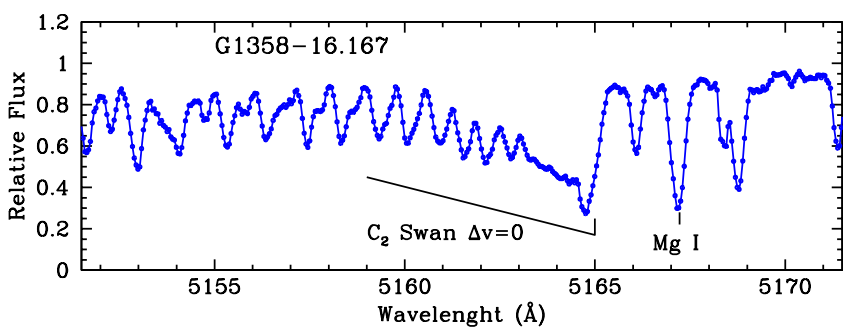

FIG. 3.- Example of a $\mathrm{C}_{2}$ Swan band in the spectrum of G1358-16.167.

process abundance indicator because (1) it is the most easily detectable in these generally mediocre spectra, and (2) the distribution of $[\mathrm{Ba} / \mathrm{Fe}]-[\mathrm{Fe} / \mathrm{H}]$ for $\omega \mathrm{Cen}$ is quite distinctive (Fig. 2a) - indeed it is unique among all star systems studied to date in the extreme overabundances of s-process elements characterizing its more metal-rich stellar population (see Fig. 11 of the Geisler et al. 2007 review). Figure 2b shows the derived $[\mathrm{Ba} / \mathrm{Fe}]-[\mathrm{Fe} / \mathrm{H}]$ pattern for the 12 GGSS stars; abundances and other data derived for these stars are given in Table 1. The quoted velocities are from the original medium-resolution spectra and have typical random errors of $\sim 10 \mathrm{~km} \mathrm{~s}^{-1}$; photometric uncertainties are about $0.01 \mathrm{mag}$. Abundance uncertainties (shown in Fig. 2b) were set by the sensitivities of $\mathrm{Fe}$ I and $\mathrm{Ba}$ II abundances to changes in stellar parameters of $\pm 100 \mathrm{~K}$ in $T_{\text {eff }}, \pm 0.3$ dex in $\log g$, and \pm 0.5 $\mathrm{km} \mathrm{s}^{-1}$ in $\xi$. As is vividly demonstrated, the Table 1 stars most likely to be kinematically associated with $\omega$ Cen (Fig. 1d) clearly follow the characteristic $\omega \mathrm{Cen}$ trend in $[\mathrm{Ba} / \mathrm{Fe}]$ versus $[\mathrm{Fe} / \mathrm{H}]$, while two stars least likely to be kinematically associated with $\omega$ Cen lie along the MW trend. The combination of kinematical consistency and possession of the hallmark barium abundance trends for the former group of stars are strong evidence that an extended part of the $\omega$ Cen tidal debris stream has been found 6

It is worth noting that within this rather small sample of $\omega$ Cen stream candidates is a carbon-rich star, G135816.167. This red giant exhibits strong $S$ wan $\mathrm{C}_{2}$ bands (e.g., at $\lambda 5165 \AA$; Fig. 3 ) and is strongly barium-enhanced. Such Crich halo giants at this modestly low metallicity are relatively rare and constitute only about $1-2 \%$ of halo giants. However, $\omega$ Cen has at least five known C-rich giants (see Table 3 in Bartkevicius 1996, which both subgiants and giants) whereas the only other globular clusters known to harbor C-rich giants are M22 (with two - as well as a spread in heavy-element abundances and s-process enrichment, similar to, but not to the degree of, $\omega \mathrm{Cen}$ ), M2 (with one), and M55 (with one Smith \& Norris (1982)). Given the likely association of most Table 1 stars to $\omega$ Cen it is not too surprising that one is found to be C-rich; this observation only strengthens the tie to their chemically peculiar parent stellar system.

Based on the $[\mathrm{Fe} / \mathrm{H}]-[\alpha / \mathrm{Fe}]$ and age-metallicity distributions for $\omega$ Cen given by Stanford et al. (2006) - adopting a 4 Gyr $\omega$ Cen age span - and the derived $[\mathrm{Fe} / \mathrm{H}]$ and 2MASS photometric data for the ten good GGSS $\omega$ Cen candidates we estimate their distances using matching Dotter et al. (2008) isochrones. With these distances we can place the stars in their Galactic planar positions relative to $\omega$ Cen and the Sun (Fig. 4). Superposed on this distribution we plot the model debris and satellite orbit from one of several $\omega$ Cen models in

\footnotetext{
${ }^{6}$ The two Table 1 stars with $\omega$ Cen chemistry and extreme $v_{b}$, off the main "green trend" in Fig. 1c, are plausibly associated with older $\omega$ Cen tidal debris wraps, shown with faint probability in Fig. 1d, or not currently part of our 0.4 Gyr-long models (see Fig. 4).
}

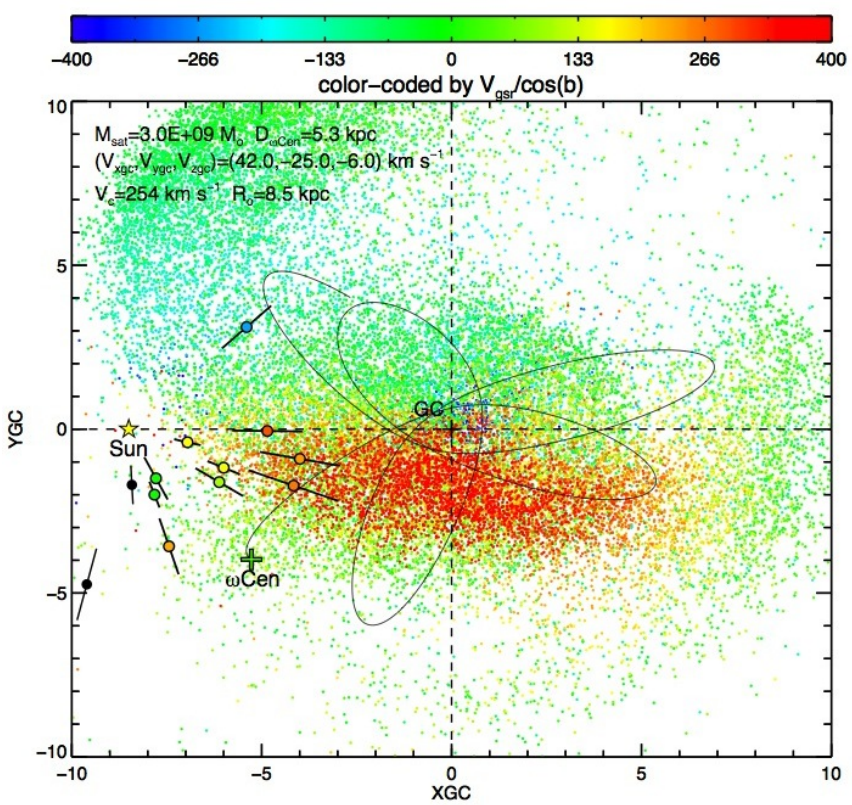

FIG. 4.- The Galactic X-Y positions of Table 1 stars with respect to the Sun $(\odot)$ and $\omega$ Cen (large green cross). Overlaid is an N-body simulation of $\omega$ Cen tidal disruption and the associated orbit over the past $0.4 \mathrm{Gyr}$ with the parameters given in the legend. The model and Table 1 stars with $\omega$ Cen-like chemistry are color-coded by $v_{b}$, which match well in general at the computed positions of the Table 1 stars. The two black points are the Table 1 stars that appear to be kinematically, spatially and chemically unassociated with $\omega$ Cen.

our grid (the example model parameters are given in the figure legend) that provide a reasonable match to the positions and RVs of the GGSS stars. This model, based on a satellite orbit with peri-/apo-Galactica limits of $(1 \mathrm{kpc}) /(7 \mathrm{kpc})$, respectively, not only demonstrates how the stars of interest very plausibly trace $\omega$ Cen debris, but also how it might be possible for $\omega$ Cen tidal debris to reach the solar neighborhood, as suggested by the various claims for this discussed in $\S 1$.

In fact, though, as stated earlier, available proper motions for the GGSS $\omega$ Cen stars are typically of low quality, the UCAC astrometry (Zacharias et al. 2010) does hint at further tantalizing connections to previous claims for nearby $\omega$ Cen debris: The GGSS $\omega$ Cen stars with the smallest derived $\left(E, L_{z}\right)$ uncertainties happen to fall in, or quite near, the " $\omega$ Cen debris expectation" box defined in $\left(E, L_{z}\right)$ by Dinescu (2002. using the same gravitational potential), whereas the weighted mean $L_{z}$ of all ten GGSS $\omega$ Cen stars, $-179 \pm 135 \mathrm{~km} \mathrm{~s}^{-1} \mathrm{kpc}$, matches extremely well the " $\omega$ Cen peak" identified within the stellar sample explored by Meza et al. (2005, their Fig. 9).

\section{SOME IMPLICATIONS}

In the GGSS sample of bright giant stars within $\sim 5 \mathrm{kpc}$ across the southern $2 / 3$ of the celestial sphere and biased toward metal-poor stars we have shown evidence that those having halo-like velocities, and particularly those with retrograde velocities, show a highly substructured, rather than random, distribution. This result is a further demonstration that even in the inner halo, at the position of the solar circle, the Galactic halo is not well-mixed, but shows the signature of multiple minor accretion events. We identify one group of stars kinematically and chemically consistent with being $\omega$ Cen debris, which we use to get a rough constraint on the $\omega$ Cen tidal stream in the inner Galaxy, a model demonstrating how $\omega$ Cen stars can reach the solar neighborhood. We have probed 
with high resolution spectroscopy less than half of the several dozen stars we associate with $\omega$ Cen $\left[l, v_{b}\right]$ features, yet all of the best candidates are found to be chemically consistent with being from $\omega$ Cen; we can project that most likely a large fraction of the fuller sample of " $\omega$ Cen" candidates are authentic. Considering that only a few dozen clearly retrograde stars are found in the southern GGSS at all (Fig. 1b-c), our results suggest that $\omega$ Cen tidal debris is a primary contributor of retrograde stars near the Sun, and likely the overwhelmingly dominant contributor in the inner two Galactic quadrants.
We gratefully acknowledge support by NASA/JPL contracts 1201670 and 1228235, and the David and Lucile Packard Foundation to build the GGSS, as well as NSF grants AST-0307851 and AST-0807945. This work would not have been possible without the generous access to the Swope telescope for development of the SIM Astrometric Grid granted by Carnegie Observatories Directors Augustus Oemler and Wendy Freedman.
Bartkevicius, A. 1996, Baltic Astronomy, 5, 217

Bekki, K. \& Freeman, K. C. 2003, MNRAS, 346, L11

Bizyaev, D., et al. 2006, AJ, 131, 1784

Carretta, E., et al. 2010, ApJ, 714, L7

Da Costa, G. S. \& Coleman, M. G. 2008, AJ, 136, 506

Dinescu, D. I. 2002, ASP Conf. Ser. 265: Omega Centauri, A Unique Window into Astrophysics, 265, 365

Dinescu, D. I., Girard, T. M., \& van Altena, W. F. 1999, AJ, 117, 1792

Dotter, A., Chaboyer, B., Jevremović, D., Kostov, V., Baron, E., \& Ferguson, J. W. 2008, ApJS, 178, 89

Eggen, O. J. 1965, in Galactic Structure, eds. A. Blaauw \& M. Schmidt, p. 111

Eggen, O. J. 1978, ApJ, 221, 881

Eggen, O. J. 1996a, AJ, 112, 1595

Eggen, O. J. 1996b, AJ, 112, 2661

Eggen, O. J. \& Sandage, A. R. 1959, MNRAS, 119, 255

Francois, P., Spite, M., Spite, F. 1988, A\&A, 191, 267

Fulbright, J. P. 2002, AJ, 123, 404

Geisler, D., Wallerstein, G., Smith, V. V., Casetti-Dinescu, D. I. 2007, PASP, 119,939

Helmi, A. \& White, S. D. M. 1999, MNRAS, 307, 495

Johnson, J. A. 2002, ApJS, 139, 219

Johnston, K. V., Spergel, D. N., \& Hernquist, L. 1995, ApJ, 451, 598

Kinman, T. D., Cacciari, C., Bragaglia, A., Buzzoni, A., \& Spagna, A. 2007, MNRAS, 375, 1381

Kotoneva, E., Innanen, K., Dawson, P. C., Wood, P. R., \& De Robertis, M. M. 2005, A\&A, 438, 957

Law, D. R., Majewski, S. R., Skrutskie, M. F., Carpenter, J. M., \& Ayub, H. F. 2003, AJ, 126, 1871

Law, D. R., Johnston, K. V., \& Majewski, S. R. 2005, ApJ, 619, 807

Lee, Y.-W., Joo, J.-M., Sohn, Y.-J., Rey, S.-C., Lee, H.-C., \& Walker, A. R. 1999, Nature, 402, 55

Leon, S., Meylan, G., \& Combes, F. 2000, A\&A, 359, 907

\section{REFERENCES}

Majewski, S. R. 1992, ApJS, 78, 87

Majewski, S. R. 1993, ARA\&A, 31, 575

Majewski, S. R. 2004, PASA, 21, 197

Majewski, S. R., et al. 2004, AJ, 128, 245

Majewski, S. R., Munn, J. A., \& Hawley, S. L. 1994, ApJ, 427, L37

Majewski, S. R., Munn, J. A., \& Hawley, S. L. 1996, ApJ, 459, L73

Majewski, S. R., Ostheimer, J. C., Kunkel, W. E., \& Patterson, R. J. 2000a, AJ, 120, 2550

Majewski, S. R., Patterson, R. J., Dinescu, D. I., Johnson, W. Y., Ostheimer, J. C., Kunkel, W. E., \& Palma, C. 2000b, Liege International Astrophysical Colloquia, 35, 619

McCarthy, J. K., Sandiford, B. A., Boyd, D., Booth, J. 1993, PASP, 105, 881 Meza, A., Navarro, J. F., Abadi, M. G., \& Steinmetz, M. 2005, MNRAS, 359,93

Mizutani, A., Chiba, M., \& Sakamoto, T. 2003, ApJ, 589, L89

Norris, J. E. \& Da Costa, G. S. 1995, ApJ, 447, 680

Patterson, R. J., et al. 2001, ASP Conf. Ser. 246: IAU Colloq. 183: Small

Telescope Astronomy on Global Scales, 246, 65

Proust, D. \& Foy, R. 1988, Ap\&SS, 145, 61

Reddy, B. E., Tomkin, J., Lambert, D. L., Allende Prieto, C. 2003, MNRAS, 340, 304

Reddy, B. E., Lambert, D. L., Allende Prieto, C. 2006, MNRAS, 367, 1329

Reid, M. J., et al. 2009, ApJ, 700, 137

Searle, L. \& Zinn, R. 1978, ApJ, 225, 357

Smith, G. H. \& Norris, J. 1982, ApJ, 254, 149

Smith, V. V., Suntzeff, N. B., Cunha, K., Gallino, R., Busso, M., Lambert, D. L., Straniero, O. 2000, AJ, 119, 1239

Stanford, L. M., Da Costa, G. S., Norris, J. E., \& Cannon, R. D. 2006, ApJ, 647, 1075

Tsuchiya, T., Dinescu, D. I., \& Korchagin, V. I. 2003, ApJ, 589, L29

Tsuchiya, T., Korchagin, V. I., \& Dinescu, D. I. 2004, MNRAS, 350, 1141

Wylie-de Boer, E., Freeman, K., \& Williams, M. 2010, AJ, 139, 636

Zacharias, N., et al. 2010, AJ, 139, 2184 
TABLE 1

GGSS STARS WITH ECHELLE SPECTROSCOPY

\begin{tabular}{|c|c|c|c|c|c|c|c|c|c|c|c|c|c|c|}
\hline Star & RA & $0.0)^{\text {Dec }}$ & $\begin{array}{l}l \\
\left({ }^{\circ}\right)\end{array}$ & $\begin{array}{l}b \\
\left({ }^{\circ}\right)\end{array}$ & $M$ & $M-T_{2}$ & $\begin{array}{c}V_{\mathrm{GSR}} \\
\left(\mathrm{km} \mathrm{s}^{-1}\right)\end{array}$ & $\begin{array}{l}\mathrm{T}_{\mathrm{eff}} \\
(\mathrm{K})\end{array}$ & $\log g$ & $\begin{array}{c}\xi \\
\left(\mathrm{km} \mathrm{s}^{-1}\right)\end{array}$ & $\begin{array}{c}{[\mathrm{Fe} / \mathrm{H}]} \\
(\mathrm{dex})\end{array}$ & $\begin{array}{l}{[\mathrm{Ba} / \mathrm{Fe}]} \\
(\mathrm{dex})\end{array}$ & $\begin{array}{l}\text { Age }^{a} \\
(G y r)\end{array}$ & $\begin{array}{c}D_{2 \mathrm{MASS}} \\
(\mathrm{kpc})\end{array}$ \\
\hline \multicolumn{15}{|c|}{ Stars least likely associated with $\omega$ Centauri } \\
\hline $\begin{array}{l}\text { G0945-22.153 } \\
\text { G1100-22.138 }\end{array}$ & $\begin{array}{l}09: 47: 51.6 \\
11: 03: 06.6\end{array}$ & $\begin{array}{l}-22: 48: 44 \\
-22: 57: 50\end{array}$ & $\begin{array}{l}257 \\
273\end{array}$ & $\begin{array}{l}23 \\
33\end{array}$ & $\begin{array}{l}13.19 \\
13.23\end{array}$ & $\begin{array}{l}1.56 \\
1.29\end{array}$ & $\begin{array}{r}121.0 \\
86.9\end{array}$ & $\begin{array}{l}4200 \\
4800\end{array}$ & $\begin{array}{l}1.5 \\
1.9\end{array}$ & $\begin{array}{l}2.0 \\
3.0\end{array}$ & $\begin{array}{l}-0.95 \pm 0.17 \\
-1.01 \pm 0.15\end{array}$ & $\begin{array}{l}+0.08 \pm 0.26 \\
-0.14 \pm 0.25\end{array}$ & $\begin{array}{l}10.8 \\
11.0\end{array}$ & $\begin{array}{l}6.1 \pm 1.3 \\
2.8 \pm 0.7\end{array}$ \\
\hline \multicolumn{15}{|c|}{ Stars most likely associated with $\omega$ Centauri } \\
\hline $\begin{array}{l}\text { G1211-05.126 } \\
\text { G1211-11.146 } \\
\text { G1232-05.60 } \\
\text { G1341+05.29 } \\
\text { G1358-16.167 } \\
\text { G1403+05.80 } \\
\text { G1443-16.11 } \\
\text { G1448-05.103 } \\
\text { G1532-05.29 } \\
\text { G2237-16.2017 }\end{array}$ & $\begin{array}{l}12: 14: 34.6 \\
12: 14: 23.6 \\
12: 35: 00.3 \\
13: 43: 31.1 \\
14: 01: 01.4 \\
14: 06: 34.1 \\
14: 45: 52.9 \\
14: 51: 32.3 \\
15: 34: 27.9 \\
22: 39: 41.9\end{array}$ & $\begin{array}{l}-05: 57: 53 \\
-11: 43: 49 \\
-06: 02: 30 \\
+05: 15: 39 \\
-17: 00: 24 \\
+05: 12: 41 \\
-17: 03: 06 \\
-06: 00: 04 \\
-05: 52: 49 \\
-16: 35: 46\end{array}$ & $\begin{array}{r}287 \\
289 \\
296 \\
335 \\
326 \\
346 \\
339 \\
349 \\
0 \\
45\end{array}$ & $\begin{array}{r}56 \\
50 \\
57 \\
65 \\
43 \\
62 \\
38 \\
46 \\
39 \\
-58\end{array}$ & $\begin{array}{l}13.22 \\
12.52 \\
12.60 \\
13.45 \\
12.55 \\
12.86 \\
12.65 \\
13.30 \\
13.12 \\
12.49\end{array}$ & $\begin{array}{l}1.81 \\
1.58 \\
1.17 \\
1.73 \\
1.47 \\
1.43 \\
1.70 \\
1.65 \\
1.52 \\
1.60\end{array}$ & $\begin{array}{r}146.3 \\
20.4 \\
23.7 \\
73.8 \\
93.4 \\
83.8 \\
219.0 \\
171.9 \\
228.8 \\
-146.9\end{array}$ & $\begin{array}{l}4075 \\
4250 \\
4800 \\
4000 \\
4400 \\
4375 \\
4375 \\
4250 \\
4500 \\
4150\end{array}$ & $\begin{array}{l}1.9 \\
2.2 \\
1.1 \\
2.0 \\
1.9 \\
2.2 \\
1.7 \\
2.1 \\
2.6 \\
1.9\end{array}$ & $\begin{array}{l}2.1 \\
1.4 \\
2.5 \\
2.0 \\
1.8 \\
2.2 \\
1.5 \\
2.4 \\
1.7 \\
2.4\end{array}$ & $\begin{array}{l}-1.07 \pm 0.23 \\
-0.82 \pm 0.14 \\
-1.93 \pm 0.20 \\
-0.71 \pm 0.14 \\
-1.29 \pm 0.20 \\
-0.76 \pm 0.19 \\
-1.90 \pm 0.27 \\
-1.20 \pm 0.23 \\
-1.51 \pm 0.20 \\
-1.79 \pm 0.19\end{array}$ & $\begin{array}{l}+0.69 \pm 0.30 \\
+0.45 \pm 0.24 \\
-0.51 \pm 0.28 \\
+0.92 \pm 0.24 \\
+0.88 \pm 0.28 \\
+0.46 \pm 0.28 \\
+0.76 \pm 0.34 \\
+0.39 \pm 0.30 \\
+0.71 \pm 0.28 \\
+0.52 \pm 0.28\end{array}$ & $\begin{array}{r}11.2 \\
10.3 \\
13.5 \\
9.9 \\
12.0 \\
10.1 \\
13.5 \\
11.7 \\
12.8 \\
13.5\end{array}$ & $\begin{array}{l}7.3 \pm 1.6 \\
3.7 \pm 0.7 \\
3.5 \pm 1.0 \\
6.8 \pm 1.1 \\
5.0 \pm 1.1 \\
3.5 \pm 0.8 \\
8.0 \pm 1.9 \\
7.8 \pm 1.7 \\
6.7 \pm 1.5 \\
9.2 \pm 1.6\end{array}$ \\
\hline
\end{tabular}

${ }^{a}$ Assumed age based on the $\omega$ Cen age-metallicity relation by Stanford et al. [2006) and our derived [Fe/H]. 